\title{
Expression changes and clinical significance of TLR-4 and IL-1 $\beta$ in serum and knee joint fluid of patients with knee osteoarthritis
}

\author{
Honggang Mao ${ }^{1}$, Yanli Zhu*2, Yan Liu ${ }^{1}$, Guodong Liu ${ }^{1}$, Fei Zhang ${ }^{1}$ \\ ${ }^{1}$ Department of Orthopaedics, the Third Affiliated Hospital of Inner Mongolia Medical University (Baogang Hospital), Baotou, \\ Inner Mongolia, China \\ ${ }^{2}$ Department of Anesthesiology, the Second Affiliated Hospital of Baotou Medical College, Inner Mongolia University of Science \\ and Technology, Baotou, Inner Mongolia, China
}

Received: August 30, 2020

Accepted: November 12, 2020 Online Published: November 30, 2020

DOI: $10.5430 /$ dcc.v7n2p25

URL: https://doi.org/10.5430/dcc.v7n2p25

\begin{abstract}
Objective: To investigate the expression changes and clinical significance of Toll-like receptor-4 (TLR-4) and interleukin-1 $\beta$ (IL-1 $\beta$ ) in the serum and knee joint fluid in patients with knee osteoarthritis (KOA).

Methods: From June 2017 to December 2018, 220 cases of patients with KOA who were admitted in Department of Orthopedics of the Third Affiliated Hospital of Inner Mongolia Medical University ("our hospital" for short) were selected as the observation group. According to the severity of KOA, they were divided into the mild subgroup $(n=98)$, the moderate subgroup $(n=80)$ and the severe subgroup $(n=42)$. In addition, 60 cases of volunteers who received the physical examination in Health Medical Center of our hospital were selected as the control group. The levels of TLR-4 and IL- $1 \beta$ in the serum and knee joint fluid were detected by enzyme-linked immunosorbent assay, in order to analyze the correlation of TLR-4 and IL-1 $\beta$ levels with the severity of KOA. Results: The levels of TLR- 4 and IL- $1 \beta$ in the serum and knee joint fluid of the observation group were significantly higher than those of the control group, and the differences were statistically significant (all $p<.05$ ). The levels of TLR- 4 and IL- $1 \beta$ in the serum and knee joint fluid of the severe subgroup were significantly higher than those of the mild subgroup and the moderate subgroup, and the levels of TLR-4 and IL- $1 \beta$ in the serum and knee joint fluid of the moderate subgroup were higher than those of the mild group. The differences were statistically significant (all $p<.05$ ). The levels of TLR- 4 and IL- $1 \beta$ in the serum and knee joint fluid were positively correlated to the severity of KOA $(r=.706, .729, .741, .715$, all $p<.05)$; in the serum, the level of TLR-4 was positively correlated to the level of IL- $\beta(r=.720, p<.05)$; in the joint fluid, the level of TLR-4 was positively correlated to the level of IL-1 $\beta(r=.736, p<.05)$.

Conclusions: The levels of TLR- 4 and IL- $1 \beta$ in the serum and knee joint fluid of KOA patients are abnormally increased, which is closely related with the severity of KOA. It can be used for clinical prediction, diagnosis and treatment of KOA.
\end{abstract}

Key Words: Knee osteoarthritis, Serum, Knee joint fluid, Toll-like receptor-4, Interleukin-1 $\beta$, Correlation

*Correspondence: Yanli Zhu; Email: nmgbgyyyy@163.com; Address: Department of Anesthesiology, the Second Affiliated Hospital of Baotou Medical College, Inner Mongolia University of Science and Technology, Baotou, Inner Mongolia, 014030, China. 


\section{INTRODUCTION}

The true nature of osteoarthritis $(\mathrm{OA})$ is articular cartilage degeneration, with limited joint motions, severely affecting the quality of life. It usually occurs in the senior population. Among all types of OA, knee osteoarthritis (KOA) accounts for $78.5 \% .^{[1,2]} \mathrm{KOA}$, which is one of clinically commonlyseen chronic inflammatory diseases, is characterized by articular cartilage degeneration, hypertrophic osteoarthropathy, sometimes osteosynovitis. Meanwhile, patients with KOA may develop some symptoms such as pain and joint deformity in the knee joints. ${ }^{[3-5]}$ With the acceleration of the aging of the population, KOA has become one of important factors leading to the disability in the senior population. Due to the lack of effective biochemical indicators used in the diagnosis, it will probably lose the best treatment opportunity if it fails to make a diagnosis of early-stage KOA. ${ }^{[6]}$

As the pathogenesis of KOA remains to be unclear, along with the lack of some effective treatment drugs, the research focus is on the pathogenesis and effective treatment methods It is generally believed that the occurrence of KOA is closely associated with multiple cytokines, such as interleukin (IL) family, and it is intended to disclose the change mechanism of tissue cells at the molecular level. In recent years, with the development of molecular biotechnology, it is gradually found that toll-like receptor (TLR) family plays an important role in the regulated function of non-specific immunity, and it can also connect specific immunity to non-specific immunity. ${ }^{[7]}$ TLR-4 shares a higher homology with IL-1 $\beta$ in cytoplasmic domain, and they are closely associated with KOA. They probably participate in the pathological course of KOA. ${ }^{[8,9]}$ It provides a new idea for the clinical exploration of the specific pathogenesis of KOA. By detecting the levels of TLR- 4 and IL- $1 \beta$ in the serum and knee joint fluid and exploring their correlation, the research is designated to provide a theoretical basis for exploring the pathogenesis of $\mathrm{KOA}$ and the treatment methods.

\section{DATA AND METHODS}

\subsection{General information}

From June 2017 to December 2018, 220 cases of KOA patients who were admitted in Department of Orthopedics of the Third Affiliated Hospital of Inner Mongolia Medical University ("our hospital" for short) were selected as the observation group, including 104 male patients and 116 female patients, aged 50-75 (62.26 \pm 5.15$)$, BMI $19.25-23.12 \mathrm{~kg} / \mathrm{m}^{2}$ $(21.25 \pm 1.36) \mathrm{kg} / \mathrm{m}^{2}$; with a course of $2-6(3.76 \pm 1.02)$ years. According to the severity of $\mathrm{KOA},{ }^{[10]}$ the observation group was subdivided into the mild subgroup $(n=98)$, the moderate subgroup $(n=80)$ and the severe subgroup $(n=42)$. In addition, 60 cases of volunteers who received the physical examination in Health Medical Center of our hospital were selected as the control group, including 26 male subjects and 34 female subjects, aged 48-77 (61.35 \pm 4.26$)$, BMI $19.10-22.89 \mathrm{~kg} / \mathrm{m}^{2}(21.07 \pm 1.17) \mathrm{kg} / \mathrm{m}^{2}$. As to the general data, there was no statistically significant difference between the two groups $(p>.05)$, and they were comparable. The research was approved by Ethics Committee of our hospital.

Inclusion criteria: 1) patients in conformity with the diagnostic standards of KOA $;^{[11]} 2$ ) patients with complete clinical data and high compliance; 3) patients and their families who volunteered and signed informed consent forms. Exclusion criteria: 1) patients with hypertension, CHD and other chronic diseases; 2) patients with a medical history of knee operation and infection; 3) patients with rheumatoid arthritis, systemic lupus erythematosus and other immune diseases; 4) patients who took glucocorticoid drugs within half a month before enrollment.

\subsection{Diagnostic standards}

All patients complied with the KOA-related standards of Guidelines for Osteoarthritis (2017 Edition) established by Chinese Orthopaedic Association: ${ }^{[11]} 1$ ) recurrent seizures of knee joint pain within one month; 2) X-ray scanning results showed narrower articular cavity, with the formation of osteophyte in the joint margin; 3) the joint fluid was clear and viscous, with the number of leukocytes less than $2 \times$ $10^{6}$ cells/L; 4) age $>40$. The duration of knee joint stiffness was less than $30 \mathrm{~min}$, with bony crepitus during the process of motion. Patients who conformed to: 1) and 2);1),3) and 4); 1)-4); were diagnosed as KOA.

The degree of cartilage injury was evaluated by use of arthroscopy: ${ }^{[11]}$ 1) Grade I, swelling on the surface of the joint; 2) Grade II, coarseness, superficial ulcer and fibrosis with the diameter less than $1 \mathrm{~cm}$ on the surface of the joint; 3 ) Grade III, deep ulcer with the diameter more than $1 \mathrm{~cm}$ on the surface of the joint, without the exposure of the subchondral bone; 4) Grade IV, with the subchondral bone exposed.

X-ray scanning was used to make a diagnosis of cartilage injury: ${ }^{[6]}$ 1) Grade I, no changes shown in the articular cavity, with tiny osteophytes; 2) Grade II, narrower articular cavity, with mild-degree osteophytes; 3) Grade III, significantly narrower articular cavity, with moderate-degree osteophytes; 4) Grade IV, significantly narrower articular cavity, with a large amount of severe-degree osteophytes.

\subsection{KOA grading standards}

Mild subgroup: Grade I cartilage injury, with KellgrenLawrence (K-L) grade ranging from 0 to I; moderate subgroup: Grade II to III cartilage injury and/or K-L Grade II; severe subgroup: Grade IV cartilage injury and/or K-L Grade 
III-IV.

\subsection{Methods}

$3 \mathrm{ml}$ of fasting peripheral venous blood was taken from each subject, placed for $20 \mathrm{~min}$, centrifuged at the rotate speed of 3,500 r/min for $10 \mathrm{~min}$ (centrifugal radius: $4 \mathrm{~cm}$ ). The supernatant was taken and placed in a $-80^{\circ} \mathrm{C}$ refrigerator for the use of measurement. Each patient was required to keep in the sitting position, after local anesthesia, $2 \mathrm{ml}$ of joint fluid was taken and placed in the Eppendorf tube, and then reserved in a $-80^{\circ} \mathrm{C}$ refrigerator for the use of measurement. Enzyme-linked immunosorbent assay was used to detect the levels of TLR-4 (Batch No.: 170427) and IL-1 $\beta$ (Batch No.: 170316) in the serum and the joint fluid. The kits were manufactured by R\&D from USA. It was required to strictly follow the instructions of kits.

\subsection{Statistical methods}

SPSS 19.0 statistical software was applied to data analysis. The levels of TLR- 4 and IL- $1 \beta$ in the serum and the joint fluid were represented by mean \pm standard deviation $(\bar{X} \pm$ s). One-way ANOVA was applied to the comparison among multiple groups, LSD test and $t$-test were used in the com- parison between two groups. The correlation of TLR-4 and IL-1 $\beta$ levels in the serum and the joint fluid to the severity of KOA was analyzed by use of Pearson or Spearman correlation analysis. The difference $p<.05$ was of statistical significance.

\section{RESULTS}

3.1 The comparison in the levels of TLR-4 and IL-1 $\beta$ in the serum and the joint fluid between two groups

The levels of TLR- 4 and IL- $1 \beta$ in the serum and the joint fluid in the observation group were higher than those in the control group (all $p<.05$ ). See Table 1 for details.

\subsection{The comparison in the levels of TLR- 4 and IL- $1 \beta$ in the serum and the joint fluid among different KOA subgroups}

The levels of TLR- 4 and IL- $1 \beta$ in the serum and the joint fluid in the severe subgroup were higher than those in the mild and the moderate subgroups, and the levels of TLR-4 and IL-1 $\beta$ in the serum and the joint fluid in the moderate subgroup were higher than those in the mild subgroup. The differences were all of statistical significance (all $p<.05$ ). See Table 2 for details.

Table 1. The comparison in the levels of TLR-4 and IL-1 $\beta$ in the serum and the joint fluid between two groups $(\bar{X} \pm \mathrm{s})$

\begin{tabular}{|c|c|c|c|c|c|}
\hline \multirow{2}{*}{ Group } & \multirow{2}{*}{$\mathbf{n}$} & \multicolumn{2}{|c|}{ Serum } & \multicolumn{2}{|c|}{ Joint Fluid } \\
\hline & & TLR-4 (ng/ml) & IL-1ß (pg/ml) & TLR-4 (ng/ml) & IL-1及 (pg/ml) \\
\hline Control Group & 60 & $8.71 \pm 2.06$ & $4.06 \pm 1.23$ & $11.89 \pm 3.05$ & $7.23 \pm 1.89$ \\
\hline Observation Group & 220 & $12.85 \pm 3.17$ & $7.86 \pm 2.35$ & $14.73 \pm 3.22$ & $9.15 \pm 2.37$ \\
\hline$t$ Value & & 9.573 & 12.072 & 6.123 & 5.791 \\
\hline$p$ Value & & .000 & .000 & .000 & .000 \\
\hline
\end{tabular}

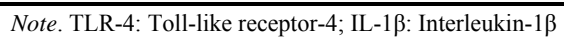

Table 2. The comparison in the levels of TLR-4 and IL- $1 \beta$ in the serum and the joint fluid among different KOA subgroups $(\bar{X} \pm \mathrm{s})$

\begin{tabular}{|c|c|c|c|c|c|}
\hline \multirow{2}{*}{ Group } & \multirow{2}{*}{$\mathbf{n}$} & \multicolumn{2}{|c|}{ Serum } & \multicolumn{2}{|c|}{ Joint Fluid } \\
\hline & & TLR-4 (ng/ml) & IL-1ß (pg/ml) & TLR-4 (ng/ml) & IL-1ß (pg/ml) \\
\hline Mild Subgroup & 98 & $9.24 \pm 2.61$ & $5.17 \pm 1.40$ & $12.63 \pm 3.17$ & $8.54 \pm 2.02$ \\
\hline Moderate Subgroup & 80 & $12.03 \pm 3.05^{*}$ & $7.13 \pm 1.98^{*}$ & $13.96 \pm 3.64^{*}$ & $9.13 \pm 0.49^{*}$ \\
\hline Severe Subgroup & 42 & $13.16 \pm 3.29^{* \#}$ & $8.21 \pm 2.53^{* \#}$ & $15.06 \pm 4.06^{* \#}$ & $9.33 \pm 0.57^{* \#}$ \\
\hline$F$ Value & & 34.470 & 46.632 & 7.723 & 6.258 \\
\hline$p$ Value & & .000 & .000 & .001 & .002 \\
\hline
\end{tabular}

Note. In comparison with the mild subgroup, ${ }^{*} p<.05$; in comparison with the moderate subgroup, ${ }^{\sharp} p<.05$. TLR-4: Toll-like receptor-4; IL-1 $\beta$ : Interleukin- $1 \beta$

\subsection{Spearman correlation analysis results}

The levels of TLR-4 and IL- $1 \beta$ in the serum and the joint fluid were positively correlated to the severity of $\mathrm{KOA}(r=$ $.706, .729, .741,0.715$, all $p<.05)$.

Published by Sciedu Press

\subsection{Pearson correlation analysis results}

The level of TLR-4 in the serum was positively correlated to the level of IL- $1 \beta$ in the serum $(r=.720, p<.05)$; the level of TLR-4 in the joint fluid was positively correlated to the 
level of IL- $1 \beta$ in the joint fluid $(r=.736, p<.05)$.

\section{Discussion}

There is still a lack of effective treatment options for KOA at present. The tissue cells in patients with KOA are stimulated by multiple injury factors, leading to the degeneration even necrocytosis of tissue cells; nevertheless, the body will generate a series of stress responses to relieve the degree of injury. ${ }^{[1]}$ It is reported that, ${ }^{[12]}$ the causes of KOA are trauma, age, abnormal immune function, overburden, familial inheritance and so on. However, the specific pathogenesis remains to be studied. Some researches have shown that, ${ }^{[8]}$ inflammatory reactions and immunological stress reactions are significant to the occurrence and development of KOA. Not only KOA is an inflammatory disease affecting all joints, but also autoimmune responses play an important role in the development of KOA. ${ }^{[13]}$

In 1985, Toll-like receptors were firstly found by Nusslein Volhard in drosophila embryos, and this type of receptors belongs to Type I transmembrane receptors, which can recognize exogenous ligands such as polysaccharide, bacterial lipoprotein and so on. ${ }^{[7-9]}$ TLR-4, as an important member of Toll family, is considered as one of Type I transmembrane receptors, which is composed of intracellular domain, extracellular domain and transmembrane domain. It can participate in the development of multiple diseases by specifically activating autoimmune responses. ${ }^{[14]}$ Located at 9q32-33, TLR-4 consists of 879 amino acids, and the extracellular domain is made up of the sequence rich in leucines. ${ }^{[15]}$ TLR4 shares a high homology with IL- $1 \beta$ in the inflammatory reaction. IL- $1 \beta$ is an important member in IL- 1 family, and it is strongly positively expressed in the cartilage tissues in KOA patients. Animal experiments and cellular tests also show that IL- $1 \beta$ is of great significance to KOA. ${ }^{[7]}$

In this research, it is found that the levels of TLR- 4 and IL- $1 \beta$ in the serum and the joint fluid in the observation group are higher than those in the control group $(p<.05)$. Meantime, with the aggravation of KOA, the levels of TLR- 4 and IL- $1 \beta$ in the serum and the joint fluid are obviously up-regulated $(p<.05)$. In addition, Spearman correlation analysis shows that the levels of TLR- 4 and IL- $1 \beta$ in the serum and the joint fluid are positively correlated to the severity of KOA $(p<$ $.05)$; it is indicated that both TLR-4 and IL- $1 \beta$ participate in the occurrence and development of KOA, which is consistent with the research results from WANG Xuezong, et al. ${ }^{[7]}$ It is because that TLR-4 signal transduction pathway can activate the release of chondrolysis enzymes and inflammatory factors (such as matrix metalloproteinases, tumor necrosis factors and IL-1) and lead to abnormal cartilage metabolic balance in KOA patients. ${ }^{[16,17]}$ The involvement of IL-1 $\beta$ in the process of KOA may be related to the following aspects: 1) the synthesis of matrix metalloproteinases: matrix metalloproteinases can degrade the protein ingredients of the extracellular matrix and lead to $\mathrm{KOA}^{\left[{ }^{[18]}\right.}{ }^{2)}$ ) the effect on improving the expression levels of tumor necrosis factors and vascular endothelial cell adhesion factors and enhancing inflammatory mediators such as CO etc.; ${ }^{[19]} 3$ ) the reduction of the synthetic amount of proteoglycans and the increase of the amount of glucolysis lead to the degradation of cartilage matrix, accelerate the apoptosis of cartilage cells and mutually participate in the occurrence of KOA; $\left.{ }^{[20]} 4\right)$ enhancing the growth speed of B cells, inhibiting the expression of collagens in cartilage cells and performing a bone resorption function; ${ }^{[21]} 5$ ) accelerating the release of prostaglandin E2 in synovial tissues and cartilage tissues, and E2 can improve the effect of IL- $1 \beta$ on degrading the cartilage; ${ }^{[22]} 6$ ) improving the pro-inflammatory effect of the collagenases. ${ }^{[8]}$

Pearson correlation analysis shows that the level of TLR-4 in the serum is positively correlated to the level of IL- $1 \beta$ in the serum $(p<.05)$; the level of TLR-4 in the joint fluid is positively correlated to the level of IL- $1 \beta$ in the joint fluid $(p<.05)$. It is indicated that TLR-4 is closely associated with IL- $1 \beta$, and it is probably due to the high homology. TLR/4Myd88/NF- $\kappa \mathrm{B}$ signaling pathway is used to activate nuclear factors, induce systemic immune response and mutually participate in the development of KOA. ${ }^{[8]}$

In conclusion, the abnormal increase in the levels of TLR-4 and IL- $1 \beta$ in the serum and the joint fluid in KOA patients is closely associated with the development of the disease. These two indicators can not only be used to predict the occurrence of the disease, but also used to monitor the development degree of KOA. In addition, they can be used as the target of clinical treatment for KOA and be worthy of clinical application.

\section{Conflicts OF InTEREST Disclosure}

The authors declare they have no conflicts of interest. 


\section{REFERENCES}

[1] Qian C, Bian X. Toll-like receptor 4 and knee osteoarthritis chondrocytes apoptosis. Journal of Jiangsu University (Medicine Edition). 2015; 25(2): 128-132.

[2] Li Y, Li Z, Ren R, et al. Research progress of knee osteoarthritis treatment. China Modern Medicine. 2019; 26(16): 24-27.

[3] Clair AJ, Kingery MT, Anil U, et al. Alterations in synovial fluid biomarker levels in knees with meniscal injury as compared with asymptomatic contralateral knees. Am J Sports Med. 2019; 47(4): 847-856. PMid:30786221. https://doi.org/10.1177/036354 6519825498

[4] Luo Q, Ji S, Li Z, et al. Effects of ultrasound therapy on the synovial fluid proteome in a rabbit surgery-induced model of knee osteoarthritis. Biomed Eng Online. 2019; 18(1): 18. PMid:30795769. https://doi.org/10.1186/s12938-019-0637-2

[5] Wu X, Tang L, Wang B, et al. Study on the Correlation of Synovial Fluid Uric Acid, Interleukin-1 $\beta$, Interleukin-6 and Tumor Necrosis Factor- $\alpha$ with Knee Osteoarthritis. West China Medical Journal. 2015; 30(10): 1862-1865.

[6] Zuo Y, Ye J, Mei X, et al. Study on the change of pro-inflammatory and anti-inflammatory factors expression in serum and synovial fluid in patients with knee osteoarthritis. China Medicine and Pharmacy, 2019, 9 (9): 217-219.

[7] Wang X, Ding D, Xue Y, et al. Role of TLR4/NF- $\kappa$ B pathway for early change of synovial membrane in knee osteoarthritis rats. China Journal of Orthopaedics and Traumatology. 2019; 32(1): 68-71.

[8] Li C. Expressions and clinical significance of toll like receptor-4 and interlukin- $\beta$ in knee osteoarthritis. Chinese Journal of Joint Surgery (Electronic Version). 2017; 11(1): 64-67. https ://doi.org/10.1 4777/uti.2016.11.1.1

[9] Liu Y, Wang B, Chen S, et al. Changes of blood biochemical indexes, hepatic fibrosis markers and inflammatory factors in liver fibrosis mice with TLR3 gene deficiency. Journal of Clinical and Experimental Medicine. 2018; 17(11): 1134-1137.

[10] $\mathrm{Su} X$, Kong Z, Zhu J, et al. The expressions and related discussion of YKL-40 and IL- $1 \beta$ in the cartilage of knee osteoarthritis. Chongqing Medicine. 2017; 46(4): 480-482.

[11] Chen L, Yang X. Serum Expression of Inflammatory Cytokines IL$1 \beta$, IL-6 and COX-2 in Osteoarthritis Patients. Chinese Journal of Clinical Medicine. 2016; 23(1): 61-62.
[12] Wang H, Wang Q, Shi X, et al. Expression and Significance of TLRs and NF- $\kappa$ B in Knee Osteoarhritic Synovial of Rats. Chinese Journal of Traditional Medical Traumatology \& Orthopedics. 2016; 24(6): 4-8.

[13] Yu Y, Chen J, Ruan Q, et al. The preliminary research on the expression of toll-like receptor4/NF- $\kappa \mathrm{B}$ in human knee osteoarthritis. Orthopaedic Biomechanics Materials and Clinical Study. 2015; 12(4): 4-7, 81 .

[14] Almeida LE, Hresko K, Sorenson A, et al. Immunohistochemical expression of TLR-4 in temporomandibular joint dysfunction. Cranio. 2019; 37(5): 323-328. PMid:29609510. https ://doi.org/10.1 $080 / 08869634.2018 .1446770$

[15] Wei S, Shen X, Li X, et al. Effects of channel-sinew micro-invasive release for the treatment of knee osteoarthritis on theTLR4/MyD88/NF$\kappa \mathrm{B}$ signal transduction pathway. China Journal of Traditional Chinese Medicine and Pharmacy. 2018; 33(10): 4637-4641. https: //doi.org/10.1166/nnl.2018.2791

[16] He J, Yan C, An F, et al. Research progress of the regulation mechanism of inflammatory factors and signaling pathways in osteoarthritis of the knee. The Chinese Journal of Clinical Pharmacology. 2019; 35(12): 1308-1311.

[17] Wang H, Wang Q, Yin Y, et al. Toll-like receptors in osteoarthritis. Journal of Changchun University of Chinese Medicine, 2016, 32 (6): 1265-1268.

[18] Liu C, Zhao L, Teng X, et al. Effects of Jintiange capsule on MMP-3, TIMP-1, IL- $1 \beta$, and TGF- $\beta 1$ levels in synovial fluid of patients with knee osteoarthritis. Chinese Journal of Osteoporosis. 2015; 21(4): 442-446.

[19] Yang L, Wang Q, Wang H, et al. Effect of Tongluozhitong Recipe on Expression of IL-1 $\beta$, HA and NO in Synovial Fluid of Human Knee Osteoarthritis. Chinese Journal of Traditional Medical Traumatology \& Orthopedics. 2017; 25(1): 1-4.

[20] Huang Q, Li J, Wen L, et al. Research progress on the inflammatory cytokines. Rheumatism and Arthritis. 2016; 5(11): 74-76, 80.

[21] Cui G, Cheng C, Shan W, et al. Effects of lactoferrin on the production of MMPs, COX-2 and PGE2 in human osteoarthritis synovial fibroblasts induced by IL- $1 \beta$. Acta Universitatis Medicinalis Anhui. 2019; 54(8): 1199-1204.

[22] Chen W, Zhou R, Wang Y, et al. Effect of Gulinggao and Its Disassembled Prescriptions on Inflammatory Cytokines and Aggrecanase of Osteoarthritis Model Rats. Journal of Traditional Chinese Medicine. 2016; 57(3): 245-249. 